\title{
Spatial structure of the color field in the SU(3) flux tube
}

\section{Baker}

Department of Physics, University of Washington, WA 98105 Seattle, USA

E-mail: mbaker4 auw. edu

\section{Paolo Cea}

INFN - Sezione di Bari, I-70126 Bari, Italy

E-mail: paolo.cea@ba.infn.it

\section{Volodymyr Chelnokov* \\ INFN - Gruppo collegato di Cosenza, I-87036 Arcavacata di Rende, Cosenza, Italy, on leave of absence from Bogolyubov Institute for Theoretical Physics of National Academy of Sciences of Ukraine \\ E-mail: volodymyr.chelnokovalnf.infn.it}

\section{Leonardo Cosmai}

INFN - Sezione di Bari, I-70126 Bari, Italy

E-mail: leonardo.cosmai@ba.infn.it

\section{Francesca Cuteri}

Institut für Theoretische Physik, Goethe Universität, 60438 Frankfurt am Main, Germany

E-mail: cuteri@th.physik.uni-frankfurt.de

\section{Alessandro Papa}

Dipartimento di Fisica, Università della Calabria, I-87036 Arcavacata di Rende, Cosenza, Italy and INFN - Gruppo collegato di Cosenza, I-87036 Arcavacata di Rende, Cosenza, Italy

E-mail: papa@cs.infn.it

\begin{abstract}
We report on the chromoelectric and chromomagnetic fields generated by a static quark-antiquark pair at zero temperature in pure gauge SU(3). From the spatial structure of chromoelectric field we extract its nonperturbative part and discuss its properties.
\end{abstract}

The 36th Annual International Symposium on Lattice Field Theory - LATTICE2018

22-28 July, 2018

Michigan State University, East Lansing, Michigan, USA.

\footnotetext{
${ }^{*}$ Speaker.
} 


\section{Introduction}

Monte Carlo simulations of the lattice theories provide a way to investigate the nonperturbative behavior of strongly interacting particles. Numerical studies of various effective models [1-23] show that quark confinement is realized by the formation of "flux tubes", in which the chromoelectric field created by a quark-antiquark pair is confined to a narrow region around the line connecting the quarks, which provides linear potential between the quarks [24-27]. The field in such flux tubes is mainly directed along the tube axis and does not change when moving along the axis far enough from the sources [10]. Any theory claiming to explain the mechanism of confinement should be able to describe the field distribution in the flux tube in good agreement with the lattice data.

We report on measurements of all the components of the chromoelectromagnetic field created by a static quark-antiquark pair in pure gauge $\mathrm{SU}(3)$ at zero temperature, for different values of quark-antiquark separation. Our results show that while the longitudinal field component is dominant, the behavior of the transverse components can be used to subtract the perturbative part of the field, getting what we identify as the confining field forming the flux tube.

\section{Lattice observables and details of measurements}

We performed our simulations for a pure SU(3) lattice gauge theory in four dimensions, with standard Wilson action. The simulations were performed using the publicly available MILC code, modified to introduce the relevant observables.

To measure the field generated by a quark-antiquark pair we use the connected correlation function $[5,6,28,29]$

$$
\rho_{W, \mu \nu}^{\text {conn }}=\frac{\left\langle\operatorname{tr}\left(W L U_{P} L^{\dagger}\right)\right\rangle}{\langle\operatorname{tr}(W)\rangle}-\frac{1}{3} \frac{\left\langle\operatorname{tr}\left(U_{P}\right) \operatorname{tr}(W)\right\rangle}{\langle\operatorname{tr}(W)\rangle}
$$

where $U_{P}$ is a plaquette in the $\mu \nu$ plane, $W$ is a Wilson loop that generates a static quark-antiquark pair, and $L$ is a Schwinger line connecting the plaquette to the Wilson loop (see Figure 1). The connected correlation function (2.1) has a naive continuum limit [6]

$$
\rho_{W, \mu \nu}^{\operatorname{conn}} \stackrel{a \rightarrow 0}{\longrightarrow} a^{2} g\left[\left\langle F_{\mu \nu}\right\rangle_{q \bar{q}}-\left\langle F_{\mu \nu}\right\rangle_{0}\right]
$$

where the subscript $q \bar{q}$ refers to the average field in presence of a quark-antiquark pair and the subscript 0 denotes average value in vacuum, which is expected to vanish. Different orientations of the plaquette correspond to the three components of the chromoelectric field $\vec{E}$, when the plaquette is time-like, and to the three components of the chromomagnetic field $\vec{B}$, when the plaquette is spatial.

To reduce fluctuations we used a smearing procedure consisting of one HYP smearing step [30] with parameters $\left(\alpha_{1}, \alpha_{2}, \alpha_{3}\right)=(1.0,0.5,0.5)$ for the links in the temporal direction, and a set of $N_{\text {sm }}$ APE smearing steps [31] for the links in spatial directions with $\alpha_{\mathrm{APE}}=0.25$.

We have performed simulations for the three different values of $\beta$, described in Table 1 . To set the physical scale a parametrization given in [32] was used, using the value $\sqrt{\sigma}=420 \mathrm{MeV}$ for the string tension. The measurements in each case were taken every 100 lattice updates, discarding a few thousands trajectories at the beginning to reach thermalization. Error analysis was made using jackknife algorithm, with varying blocking size. 


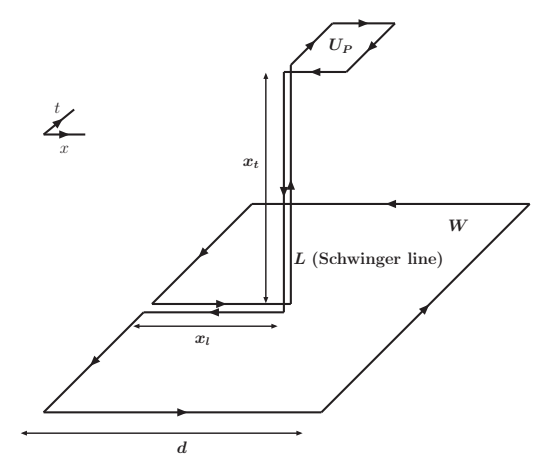

Figure 1: The connected correlator given in Eq. (2.1) between the plaquette $U_{P}$ and the Wilson loop (subtraction in $\rho_{W, \mu v}^{\text {conn }}$ not explicitly drawn).

\begin{tabular}{|c|c|c|c|c|}
\hline \hline$\beta$ & lattice & $d[\mathrm{fm}]$ & statistics & smearing steps \\
\hline 6.370 & & $0.951(11)$ & 5300 & 100 \\
6.240 & $48^{4}$ & $1.142(15)$ & 21000 & 100 \\
6.136 & & $1.332(20)$ & 84000 & 120 \\
\hline \hline
\end{tabular}

Table 1: Summary of the simulation parameters.

\section{Measurement results and Coulomb field subtraction}

At each value of $\beta$ we measured all six components of the fields at the set of displacements $\left(x_{l}, x_{t}\right)$ from the static quark (see Figure 1). The equivalence of the two choices of the direction of $x_{t}$ perpendicular to $x_{l}$, coming from the rotational symmetry of the system, was explicitly checked numerically. In what follows, the $x$ axis is chosen along the quark-antiquark line, and the $y$ axis along the direction of transverse displacement.

The three components of the chromomagnetic field $\vec{B}$ are compatible with zero within errors. The values of the three components of the chromoelectric field $\vec{E}$ at $\beta=6.370$ as a function of the displacements $x_{l}$ and $x_{t}$ are shown on the left in Figure 2. The transverse components of the chromoelectric field can be nicely described as a Coulomb-like field $\vec{E}^{C}$

$$
\vec{E}^{C}\left(\vec{r} ; Q, R_{0}\right)=Q\left(\frac{\vec{r}_{1}}{\max \left(r_{1}, R_{0}\right)^{3}}-\frac{\vec{r}_{2}}{\max \left(r_{2}, R_{0}\right)^{3}}\right)
$$

where $Q$ is an effective charge, $\vec{r}_{1}$ and $\vec{r}_{2}$ are vectors from the positions of the static quark and antiquark, respectively, to the point $\vec{r}$ where the field is measured. Since the field components are probed by a plaquette, we consider $\vec{r}$ to be the radius vector to the center of this plaquette. One important consequence of this is that the field $E_{z}$ is measured at points that are a half lattice spacing away from the $x y$ plane, so $E_{z}^{C}$ is not equal to zero. Finally $R_{0}$ is an effective radius of the charge, which we introduced to try to describe the behavior of the field on distances less then three lattice spacing from the quarks - where the field $\vec{E}^{C}$ fails to give a good description of the measured field.

We performed a series of fit of the two transverse field components $E_{y}$ and $E_{z}$ at different planes transverse to the line connecting the two quarks, treating the effective charge $Q$ and effective radius $R_{0}$ as the fit parameters. The results of these fits are summarized in Table 2; the errors of 

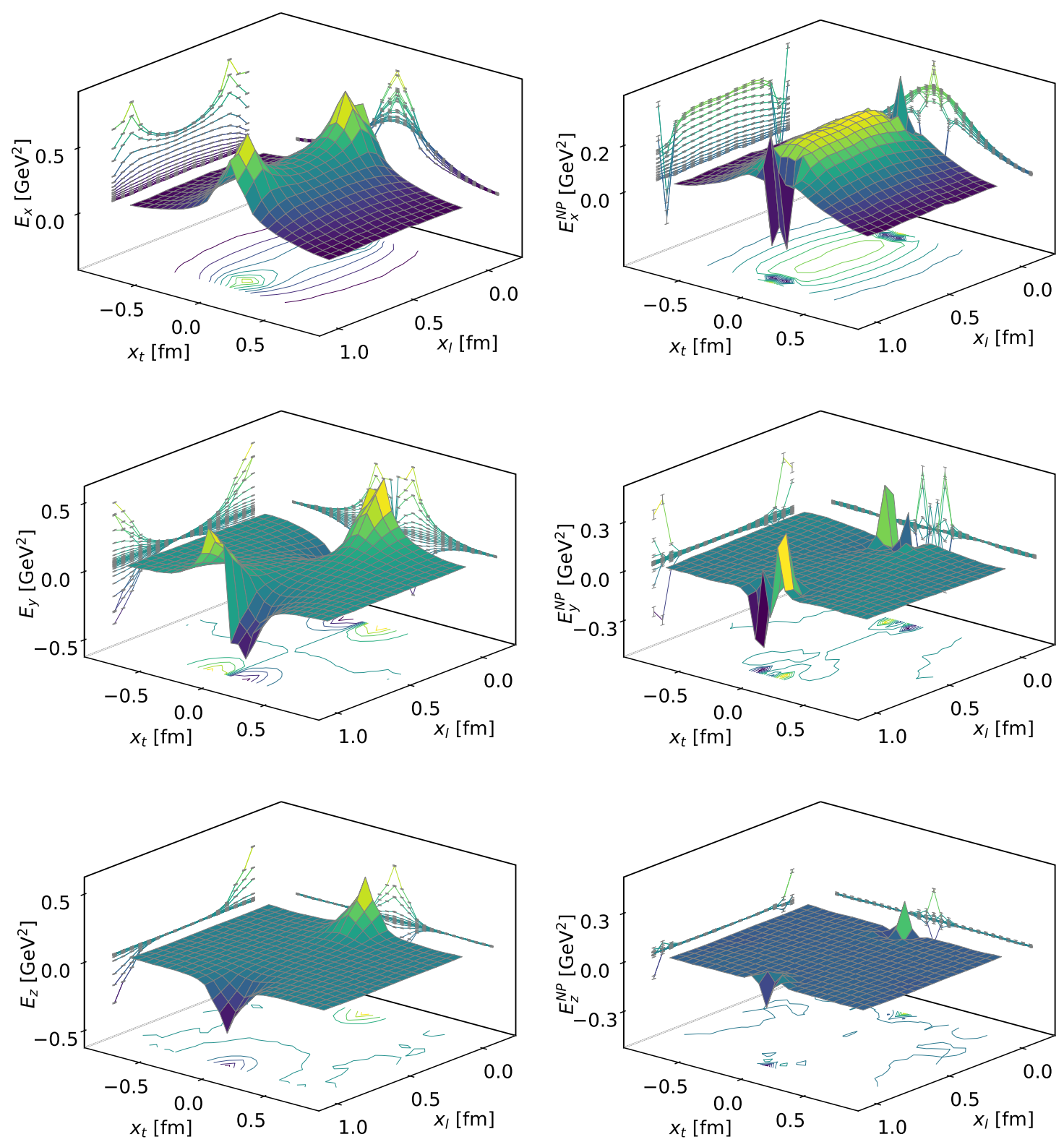

Figure 2: Surface and contour plots for the three components of the chromoelectric field (left) and its nonperturbative part (right) at $\beta=6.370$ and $d=0.951 \mathrm{fm}$.

fit parameters were obtained from a comparison of the best fit parameters for different transverse planes at which we managed to obtain a good fit.

In the further analysis we consider the chromoelectric field $\vec{E}$ to consist of two parts - nonperturbative part $\vec{E}^{N P}$ and the perturbative Coulomb-like part $\vec{E}^{C}$,

$$
\vec{E}(\vec{r})=\vec{E}^{N P}(\vec{r})+\vec{E}^{C}\left(\vec{r} ; Q, R_{0}\right)
$$

taking the values of $Q$ and $R_{0}$ from the results of our previous fitting analysis. In this way we extract the nonperturbative contribution to the chromoelectric field. This procedure makes the 


\begin{tabular}{|c|c|c|c|c|}
\hline \hline$\beta$ & $Q$ & $R_{0} / a$ & $R_{0}[\mathrm{fm}]$ & $d[\mathrm{fm}]$ \\
\hline 6.370 & $0.278(4)$ & $1.920(14)$ & $0.1142(16)$ & $0.951(11)$ \\
6.240 & $0.289(11)$ & $1.92(3)$ & $0.1367(29)$ & $1.142(15)$ \\
6.136 & $0.305(14)$ & $2.15(7)$ & $0.179(6)$ & $1.332(20)$ \\
\hline \hline
\end{tabular}

Table 2: Values of the fit parameters extracted from Coulomb fits of the transverse components of the chromoelectric field.

nonperturbative part purely longitudinal, apart from the small region of radius $\sim R_{0}$ around the static quarks. The three components of the obtained nonperturbative field $\vec{E}^{N P}$ for $\beta=6.370$ are shown on the right in Figure 2. As can be seen from these plots the longitudinal component of the field after subtraction $E_{x}^{N P}$ is much more stable under changes of the $x_{l}$ coordinate, making it useful to extract the shape of the flux tube for small distances between quarks. This fact is more clear when looking at transverse and longitudinal sections of the field shown in Figure 3. We remark that while all the plots are given for the $\beta=6.370$ case the results are qualitatively the same for other $\beta$ values.
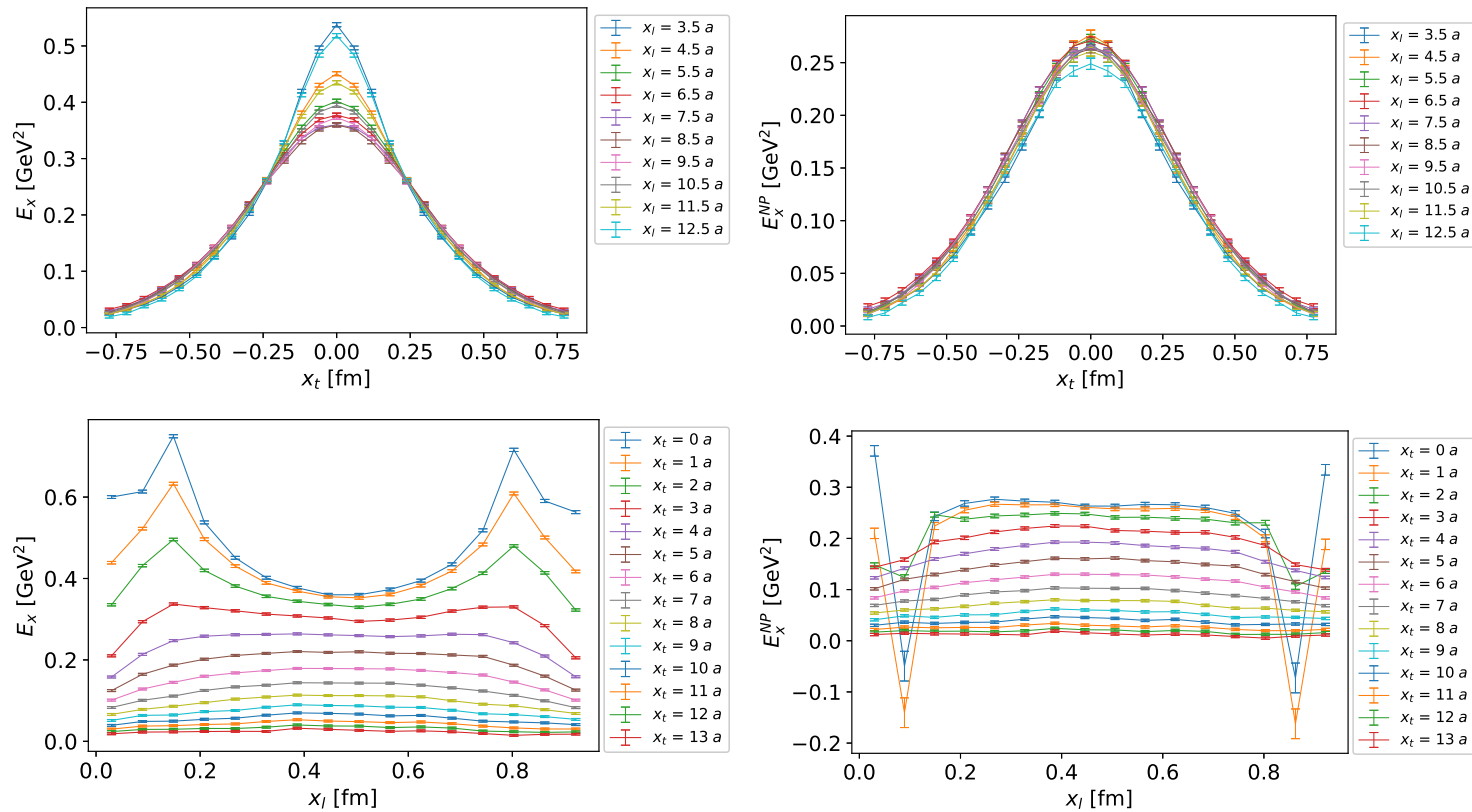

Figure 3: Transverse (top) and longitudinal (bottom) sections of the longitudinal component of the full chromoelectric field (left) and its nonperturbative part (right) at $\beta=6.370$ and $d=0.951 \mathrm{fm}$.

\section{Conclusions}

We have performed measurements of the six components of the chromoelectromagnetic field created by a static quark-antiquark pair in the four-dimensional pure gauge SU(3) theory at zero temperature.

The measured chromomagnetic field is compatible with zero. The transverse components of the chromoelectric field decay fast with the distance from the two quarks, and can be described by 
the Coulomb-like behavior. Extracting the parameters of the Coulomb law from the transverse field components, and subtracting the longitudinal component of the resulting Coulomb-like field from the measured longitudinal chromoelectric field, yields, in a model-independent way, the nonperturbative chromoelectric field forming the confining flux tube, thereby removing (to a certain degree) the perturbative short-range corrections, existing in the original field. We believe this approach can be straightforwardly applied also at nonzero temperature and/or within a theory with dynamical quarks.

\section{Acknowledgements}

This investigation was in part based on the MILC collaboration's public lattice gauge theory code. See http://physics.utah.edu/ detar/milc.html. Numerical calculations have been made possible through a CINECA-INFN agreement, providing access to resources on MARCONI at CINECA. AP, LC, PC, VC acknowledge support from INFN/NPQCD project. FC acknowledges support from the German Bundesministerium für Bildung und Forschung (BMBF) under Contract No. 05P1RFCA1/05P2015 and from the DFG (Emmy Noether Programme EN 1064/2-1). VC acknowledges financial support from the INFN HPC_HTC project.

\section{References}

[1] M. Fukugita and T. Niuya, Distribution of chromoelectric flux in SU(2) lattice gauge theory, Phys. Lett. B132 (1983) 374.

[2] J. E. Kiskis and K. Sparks, Illustrated study of flux patterns in SU(2) lattice gauge theory, Phys. Rev. D30 (1984) 1326.

[3] J. W. Flower and S. W. Otto, The field distribution in SU(3) lattice gauge theory, Phys. Lett. B160 (1985) 128.

[4] J. Wosiek and R. W. Haymaker, On the space structure of confining strings, Phys. Rev. D36 (1987) 3297.

[5] A. Di Giacomo, M. Maggiore and S. Olejnik, Evidence for flux tubes from cooled QCD configurations, Phys. Lett. B236 (1990) 199.

[6] A. Di Giacomo, M. Maggiore and S. Olejnik, Confinement and Chromoelectric Flux Tubes in Lattice QCD, Nucl. Phys. B347 (1990) 441.

[7] P. Cea and L. Cosmai, Lattice investigation of dual superconductor mechanism of confinement, Nucl. Phys. Proc. Suppl. 30 (1993) 572.

[8] Y. Matsubara, S. Ejiri and T. Suzuki, The (dual) Meissner effect in SU(2) and SU(3) QCD, Nucl. Phys. Proc. Suppl. 34 (1994) 176 [hep-lat/9311061].

[9] P. Cea and L. Cosmai, Dual Meissner effect and string tension in SU(2) lattice gauge theory, Phys. Lett. B349 (1995) 343 [hep-lat/9404017].

[10] P. Cea and L. Cosmai, Dual superconductivity in the SU(2) pure gauge vacuum: A Lattice study, Phys. Rev. D52 (1995) 5152 [hep-lat/9504008].

[11] G. S. Bali, K. Schilling and C. Schlichter, Observing long color flux tubes in SU(2) lattice gauge theory, Phys. Rev. D51 (1995) 5165 [hep-lat/ 9409005 ]. 
[12] P. Skala, M. Faber and M. Zach, Magnetic monopoles and the dual London equation in SU(3) lattice gauge theory, Nucl. Phys. B494 (1997) 293 [hep-lat/9603009].

[13] R. W. Haymaker and T. Matsuki, Model independent approach to studies of the confining dual Abrikosov vortex in SU(2) lattice gauge theory, Phys. Rev. D75 (2007) 014501 [hep-lat/0505019].

[14] A. D’Alessandro, M. D'Elia and L. Tagliacozzo, Dual superconductivity and vacuum properties in Yang-Mills theories, Nucl. Phys. B774 (2007) 168 [hep-lat/ 0607014 ].

[15] M. S. Cardaci, P. Cea, L. Cosmai, R. Falcone and A. Papa, Chromoelectric flux tubes in QCD, Phys. Rev. D83 (2011) 014502 [1011. 5803 ].

[16] P. Cea, L. Cosmai and A. Papa, Chromoelectric flux tubes and coherence length in QCD, Phys. Rev. D86 (2012) 054501 [1208.1362].

[17] P. Cea, L. Cosmai, F. Cuteri and A. Papa, Flux tubes and coherence length in the SU(3) vacuum, in Proceedings, 31 st International Symposium on Lattice Field Theory (Lattice 2013), vol. LATTICE2013, p. 468, 2013, 1310.8423.

[18] P. Cea, L. Cosmai, F. Cuteri and A. Papa, Flux tubes in the SU(3) vacuum: London penetration depth and coherence length, Phys. Rev. D89 (2014) 094505 [1404.1172].

[19] P. Cea, L. Cosmai, F. Cuteri and A. Papa, London penetration depth and coherence length of SU(3) vacuum flux tubes, PoS LATTICE2014 (2014) 350 [1410 . 4394].

[20] N. Cardoso, M. Cardoso and P. Bicudo, Inside the SU(3) quark-antiquark QCD flux tube: screening versus quantum widening, Phys. Rev. D88 (2013) 054504 [1302 .3633].

[21] M. Caselle, M. Panero, R. Pellegrini and D. Vadacchino, A different kind of string, JHEP 01 (2015) $105[1406.5127]$.

[22] P. Cea, L. Cosmai, F. Cuteri and A. Papa, Flux tubes in the QCD vacuum, Phys. Rev. D95 (2017) $114511[1702.06437]$.

[23] E. Shuryak, Are there flux tubes in quark-gluon plasma?, 1806.10487.

[24] M. Bander, Theories of quark confinement, Phys. Rept. 75 (1981) 205.

[25] J. Greensite, The confinement problem in lattice gauge theory, Prog. Part. Nucl. Phys. 51 (2003) 1 [hep-lat/0301023].

[26] G. Ripka, Confinement of quarks in dual superconductor models, AIP Conf. Proc. 775 (2005) 262.

[27] Y. A. Simonov, What is the confinement mechanism in QCD?, 1804.08946.

[28] D. S. Kuzmenko and Y. A. Simonov, Field distributions in heavy mesons and baryons, Phys. Lett. B494 (2000) 81 [hep-ph/ 0006192$].$

[29] A. Di Giacomo, H. G. Dosch, V. I. Shevchenko and Y. A. Simonov, Field correlators in QCD: Theory and applications, Phys. Rept. 372 (2002) 319 [hep-ph/ 0007223$].$

[30] A. Hasenfratz and F. Knechtli, Flavor symmetry and the static potential with hypercubic blocking, Phys. Rev. D64 (2001) 034504 [hep-lat/ 010302 9].

[31] M. Falcioni, M. Paciello, G. Parisi and B. Taglienti, Again on su(3) glueball mass, Nucl. Phys. B251 (1985) 624 .

[32] R. G. Edwards, U. M. Heller and T. R. Klassen, Accurate scale determinations for the Wilson gauge action, Nucl. Phys. B517 (1998) 377 [hep-lat/9711003]. 